\title{
Angiosarcoma of the Prostate Gland following Brachytherapy for Prostatic Adenocarcinoma
}

\author{
Arjun Gupta $^{\mathrm{a}}$ Mrinal M. Patnaik ${ }^{\mathrm{b}}$ Harris V. Naina ${ }^{\mathrm{c}}$ \\ aDepartments of Internal Medicine, University of Texas Southwestern Medical Center, Dallas, Tex.; ${ }^{b}$ Department of Hematology \\ Oncology, Mayo Clinic, Rochester, Minn.; 'Hematology Oncology, University of Texas Southwestern Medical Center, Dallas, Tex., USA
}

\section{Key Words}

Sarcoma • Prostate cancer • Radiation-induced cancer • Prostate biopsy

\begin{abstract}
Prostatic adenocarcinoma is the most common cancer in men, but only a handful of cases of prostatic angiosarcoma have been reported in the literature. Prior radiation therapy for prostatic adenocarcinoma has been hypothesized to be a risk factor for angiosarcoma. The increasing practice of prostate cancer screening and the use of radiation therapy for management of prostatic adenocarcinoma will likely lead to more cases of prostatic angiosarcoma. Diagnosis is made by tissue sampling. Optimal management of these aggressive tumors remains to be defined and outcomes are poor with a high 1-year mortality. Primary care physicians and urologists should be aware of this rare entity and refer these patients to specialist centers where they can be managed by a multidisciplinary team. We report a case of angiosarcoma of the prostate gland diagnosed in a male presenting with lower urinary tract symptoms 5 years after brachytherapy for prostate adenocarcinoma.

Copyright $\odot 2014$ S. Karger AG, Basel
\end{abstract}

\section{Introduction}

Adenocarcinoma of the prostate gland represents the most common non- dermatological malignancy in males. Sarcomas, particularly angiosarcomas, of the prostate gland on the other hand are very rarely reported. A recent review identified only 14 cases of prostatic angiosarcoma in the medical literature [1]. Angiosarcomas have been associated with exposure to radiation and chemical agents such as thorium, arsenic and vinyl chloride. These tumors are generally aggressive and frequently invade local structures and metastasize. Although surgical resection is the mainstay of therapy, management of prostatic angiosarcomas presents a problem owing to extensive local advancement, angioinvasion, and involvement of vital structures.

We present a case of angiosarcoma of the prostate gland diagnosed in a male presenting with lower urinary tract symptoms 5 years after brachytherapy for prostate adenocarcinoma.

\section{Case Report}

A 71-year-old man presented with 2 weeks history of gross hematuria and urinary frequency. Five years prior, elective screening demonstrated an elevated prostate- specific antigen (PSA) level of $4 \mathrm{ng} / \mathrm{ml}(0-3.0 \mathrm{ng} / \mathrm{ml})$. A prostate biopsy was consistent with prostatic adenocarcinoma (Gleason score $3+3$ ). He was treated with permanent interstitial brachytherapy, following which his PSA was undetectable. 


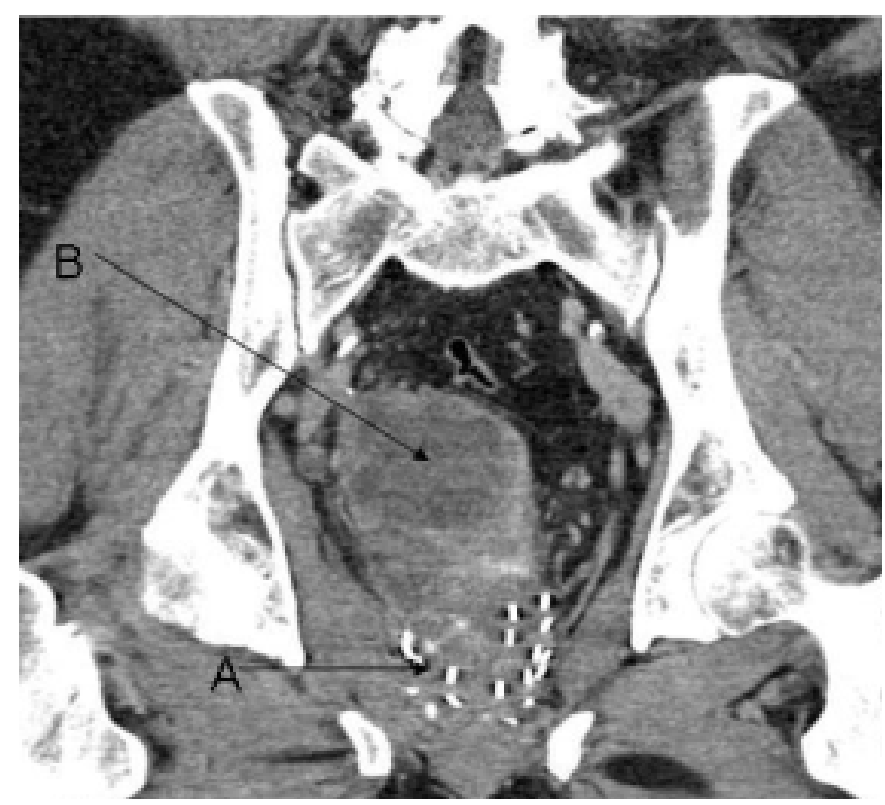

Fig. 1. CT scan of the abdomen and pelvis demonstrating the brachytherapy seeds in the prostate (A), and the large necrotic mass involving the right posterolateral side of the urinary bladder (B).

With his current visit, physical examination revealed an enlarged prostate on rectal exam. A CT urogram, demonstrated a 6.1 $\times 5.3 \mathrm{~cm}$ partially necrotic mass involving the posterolateral right side of the urinary bladder, extending beyond the lumen of the bladder into the perivesical fat (fig. 1). The CT scan also revealed enlarged perivesical and right iliac lymph nodes. Urodynamic testing showed bladder outlet obstruction without signs of neurological damage. He underwent cystoscopy, which demonstrated a necrotic tumor involving the prostatic fossa of the urethra and bladder neck. A biopsy from the mass was suggestive of grade 4/4 angiosarcoma, identified using histopathology and immunostaining (fig. 2).

The patient had no history of exposure to chemicals, including vinyl chloride, arsenic, diphenols, or thorium dioxide. He underwent radical cystoprostatectomy with pelvic lymph node dissection and Ileal conduit urinary diversion. The resected specimen showed grade $4 / 4$ angiosarcoma with epithelioid features forming a mass $(8.4 \times 7.5 \times 3.5 \mathrm{~cm})$ involving the anterior and right lateral walls, and trigone of the urinary bladder, right seminal vesicle, and the entire prostate. Surgical margins were negative.

Metastatic angiosarcoma was found in iliac, obturator, and hypogastric lymph nodes. Excisional biopsies from the peritoneum and neurovascular bundle did not show any evidence of metastatic angiosarcoma.

A PET CT scan revealed no evidence of distant metastasis. He received a total of 3 cycles of adjuvant weekly paclitaxel on a 3-week on and 1-week off schedule. However, a follow-up CT scan showed an increase in size of retrocaval lymph nodes consistent with progressive angiosarcoma. His chemotherapy was changed to once a month liposomal doxorubicin. After 3 cycles, he again demonstrated progression of his disease in the pelvic lymph nodes and his chemotherapy regimen was again changed to ifosfamide and etoposide, which he is receiving.

\section{Discussion}

Sarcoma of the prostate constitutes less than $0.1 \%$ of all prostatic malignancies. Leiomyosarcoma is the most common histological type in adults, whereas rhabdomyosarcoma is more common in pediatric patients [2]. Angiosarcoma of the prostate is very rare, and only 14 cases have been reported in the literature to date [1]. Angiosarcoma of the prostate following external beam radiation is anecdotally reported in the literature [3]. Factors known to predispose to angiosarcoma include prior radiation treatment, chronic lymphedema, and chemical exposure to vinyl chloride, thorium dioxide, and arsenic. Increased risk of soft-tissue sarcomas has been reported after adjuvant radiotherapy for breast cancer using population-based cancer registries, especially 5 years after radiation therapy [4]. In considering the diagnosis of radiation-induced sarcoma, Cahan et al. [5] elucidated the following criteria: 1) the sarcoma should arise in the area previously subjected to radiation; 2) a latent period (in years) must exist between the time of radiation and development of the sarcoma; 3) the sarcoma must be confirmed histologically. Our case developed prostate angiosarcoma 5 years after receiving radiation therapy and satisfies these criteria. A more recent review did not reveal a strong association between radiation therapy and prostatic angiosarcoma but did not rule out the possibility [6].

The wide availability of PSA testing has revolutionized prostate cancer screening. This has resulted in earlier prostate cancer detection and an increase in diagnostic and therapeutic interventions [7]. Recent studies have also demonstrated an increasing trend in the utilization of brachytherapy for the treatment of early stage prostate cancer; hence the importance in recognizing this rare but serious complication.

Campschroer et al. [1] reviewed 14 cases of angiosarcoma of prostate. Mean age was 62 years. Presenting complaints included lower urinary tract symptoms, gross hematuria and pelvic pain. On physical examination, the prostate can be enlarged, tender, or unremarkable. Diagnosis is usually made upon prostate biopsy, while serum PSA levels are not diagnostic. Abnormal, pleomorphic, malignant endothelial cells are the hallmark of angiosarcoma. Angiosarcoma's typically express endothelial markers, including factor VIII, CD34, CD31, Ulex euro- 

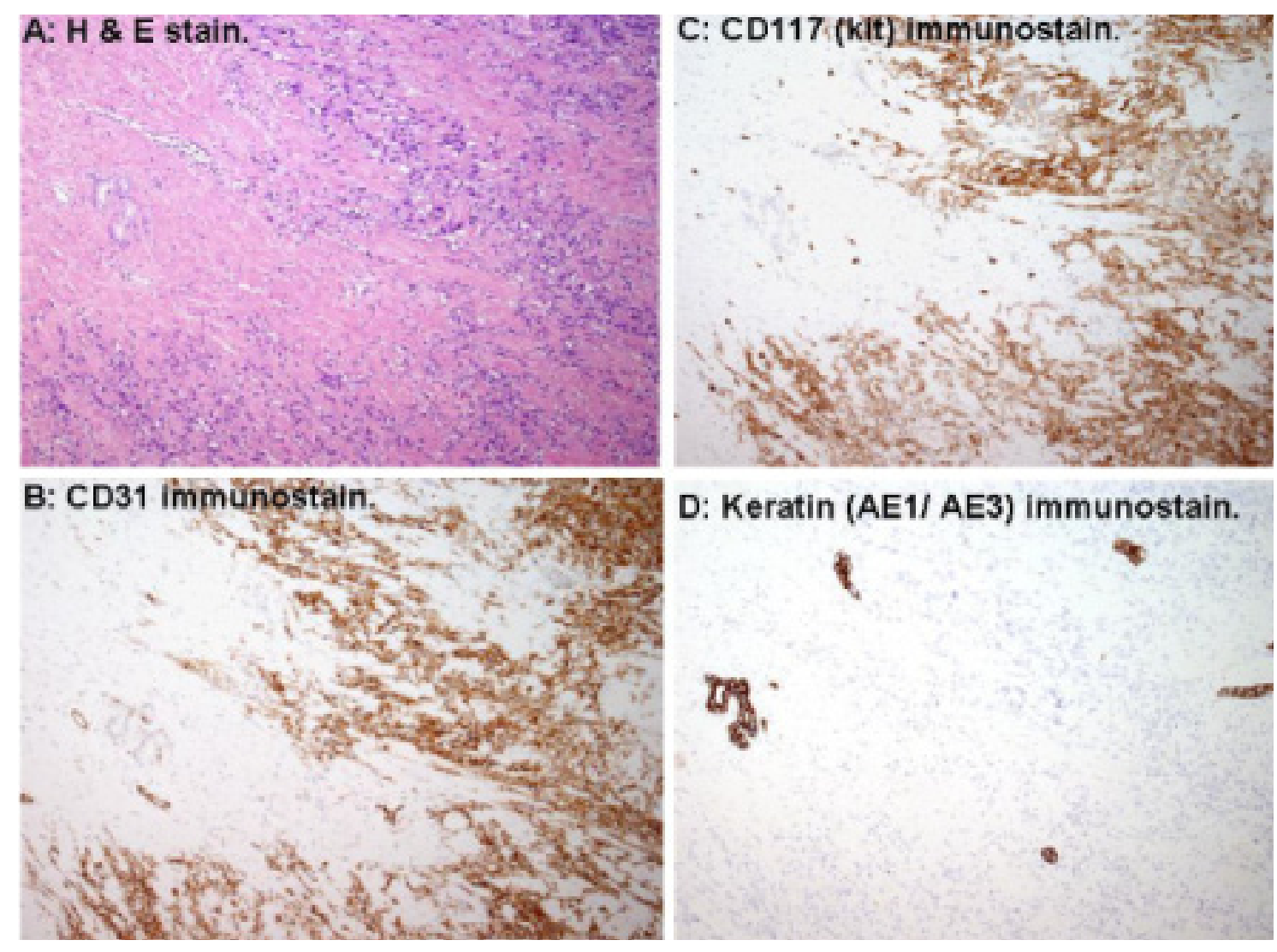

Fig. 2. A $100 \times \mathrm{H} \& \mathrm{E}$ stain demonstrating benign duct epithelium on left and undifferentiated spindled prostatic angiosarcoma on right. Immunostains performed on the tumor cells showed strong and diffuse reactivity with antibodies to FLI-1, CD31 (B 100× CD31 immunostain), and CD117 [kit] (C 100× CD117 immunostain). These cells did not react with antibodies to CD34 and a variety of keratins (OSCAR, keratin AE1/AE3, CAM 5.2, 7, 20, and 34betaE12) (D 100× keratin stain highlighting only benign prostatic duct epithelium), PSA, prostatic acid phosphatase, actin, desmin, or thyroid transcription factor.

paeus agglutinin 1, and vascular endothelial growth factor (VEGF). Immunohistochemistry is an important part of the diagnosis [8], and biopsies from our patient were positive for several of the typical angiosarcoma markers (fig. 2).

The current treatment options for prostatic angiosarcoma are limited, with surgery being the main curative modality. No randomized trials exist and recommendations are limited on a small body of evidence mostly limited to case reports. Negative surgical margins are hard to achieve given the multifocal and aggressive nature of disease [9]. Radiation therapy is generally avoided given the epidemiological association between radiation and soft-tissue sarcoma. Adjuvant chemotherapy has shown benefit over unimodal therapeutic strategy. Taxanes are often used given their anti-angiogenic nature. Novel vascular-targeting agents such as bevacizumab (anti-VEGF antibody), axitinib (VEGF-receptor tyrosine kinase inhibitor) and vadimezan (vascular-disrupting agent) are being evaluated in the management of angiosarcoma in laboratory models [10]. Our patient had locally advanced cancer with negative surgical margins after radical cystoprostatectomy, and received multiple chemotherapeutic agents; unfortunately his disease is still not cured. A high 1-year mortality has been reported and our report highlights the bad outcome associated with prostatic angiosarcoma [1].

Primary care physicians and urologists should be aware of this rare entity and refer these patients to specialist centers where they can be managed by a multidisciplinary team that includes an urologist, medical oncologist, and surgical oncologist or enrolled in a clinical trial. 


\section{References}

1 Campschroer T, van der Kwast TH, Jonges GN, Lock MT: Angiosarcoma of the prostate: a more frequent finding in the future owing to radiotherapy? A literature review with treatment implications based on a case report. Scand J Urol 2014;48:420-425.

$\checkmark 2$ Sexton WJ, Lance RE, Reyes AO, Pisters PW, Tu SM, Pisters LL: Adult prostate sarcoma: the M. D. Anderson Cancer Center Experience. J Urol 2001;166:521-525.

-3 Chandan VS, Wolsh L: Postirradiation angiosarcoma of the prostate. Arch Pathol Lab Med 2003;127:876-878.
4 Huang J, Mackillop WJ: Increased risk of soft tissue sarcoma after radiotherapy in women with breast carcinoma. Cancer 2001;92: 172-180.

5 Cahan WG, Woodard HQ, Higinbotham NL, Stewart FW, Coley BL: Sarcoma arising in irradiated bone: report of eleven cases. 1948. Cancer 1998;82:8-34.

-6 Khaliq W, Meyer CF, Uzoaru I, Wolf RM, Antonarakis ES: Prostate angiosarcoma: is there any association with previous radiation therapy? BJU Int 2012;110(11 Pt C):E819825.

7 Shariat SF, Scardino PT, Lilja H: Screening for prostate cancer: an update. Can J Urol 2008;15:4363-4374.
8 Ohsawa M, Naka N, Tomita Y, Kawamori D, Kanno H, Aozasa K: Use of immunohistochemical procedures in diagnosing angiosarcoma. Evaluation of 98 cases. Cancer 1995;75:2867-2874.

9 Fury MG, Antonescu CR, Van Zee KJ, Brennan MF, Maki RG: A 14-year retrospective review of angiosarcoma: clinical characteristics, prognostic factors, and treatment outcomes with surgery and chemotherapy. Cancer J 2005; 11:241-247.

10 Young RJ, Woll PJ, Staton CA, Reed MW, Brown NJ: Vascular-targeted agents for the treatment of angiosarcoma. Cancer Chemother Pharmacol 2014;73:259-270. 